UDC 621.3

DOI: $10.15587 / 2706-5448.2021 .233947$

Article type «Reports on Research Projects»

\title{
Liubomyr Duda
}

DEVELOPING OF THE METHOD FOR
OPTIMIZING THE PERFORMANCE
OF ARCHITECTRE-INDEPENDENT
HARDWARE PLATFORMS

The object of research is the Raspberry Pi single-board computer. The work examines the optimization of architecture-independent hardware platforms using its example. The research is based on an integrated scientific approach based on a system-analytical, structural-functional, empirical and typological approach. It is emphasized that the entire Raspberry Pi line uses APM-architecture processors. The genesis of Raspberry Pi is given, the parameters of the last build are determined. It is noted that the latest version is dated November 2020. It is equipped with wireless WiFi and Bluetooth modules (2×USB 3.0 and $1 \times$ USB 2.0 ports type A, 5.0, BLE), which expand the boundaries of mini-PC application in the field of Ethernet technologies and has a frequency of $1.8 \mathrm{GHz}$. The appearance of one of the popular Raspberry Pi B+boards has been formed, with the separation of the main blocks. The basic principles of improving the performance of the Raspberry Pi single board computer are determined, each of which is based on a specific mechanism. The first is the addition of ZRAM as a compressed random access memory block device. The principle of ZRAM operation is described, the mechanism for activating ZRAM on the Raspberry Pi is given. To improve the performance of the Raspberry Pi single board computer, the use of an NVMe disk is justified. It is emphasized that the NVMe disk is reliable and has a high data transfer rate. Connecting it to the Raspberry Pi single board computer is the optimal solution to improve performance. The tuning sequence is presented, the numerical result of the NVMe disk operation based on the Raspberry Pi single-board computer is proposed. It is proposed, as a principle to improve performance, the installation of an ICE Tower CPU based on Raspberry Pi. It is noted that the ICE Tower CPU is a cooling system that is designed to cool the Raspberry Pi. The principles of tuning ICE Tower CPU and the result of fluctuations in temperature components using the rpimonitor are described. As part of the study, performance improvements were obtained from $26 \%$ to $34 \%$, which is mainly in line with the expected theoretical improvement of $34 \%$.

Keywords: Raspberry Pi single-board computer, ARM architecture, bandwidth, architecture-independent platform, ICE Tower CPU.

\section{Introduction}

Single-board computers using operating systems based on the Linux kernel are a powerful enough tool for solving the problems of both individual users and large enterprises. But manufacturers often deliberately curtail the capabilities of these machines, using pre-programmed factory settings and unproductive hardware components. So, the development of methods for optimizing such machines is relevant. In this paper, optimization will be considered using the example of a single-board computer Raspberry Pi. The Raspberry Pi board is poorly suited for networking tasks and inconvenient in terms of USB functionality.

The Raspberry Pi has an SMSC LAN9514 chip that connects to the SoC with a single USB channel, acting as a USB-to-Ethernet adapter and USB hub at the same time.
Thus, Ethernet and USB work on the same channel and compete with each other, which is contrary to the typical use of NAS, where something is downloaded over the network and saved to a USB stick.

Over the years, upgrades have reached a maximum of $40 \mathrm{MB} / \mathrm{s}$ for raw speed and $20 \mathrm{MB} / \mathrm{s}$ if transferring to a USB device.

Scientists from different countries of the world have been developing scientific ideas to improve the performance of the Raspberry Pi single-board computer and its structure and application mechanisms for many years. In [1], the features of the structure and operation of the Raspberry Pi microcomputer (MC) are considered. On the basis of a comparative analysis of three popular platforms, the choice of this MC was justified for solving the assigned tasks. Scientists have selected equipment for the development of an intelligent information system. 
An analysis of the possibilities of using Raspberry singleboard computers in teaching distribution and parallel computing was made by the author of [2]. The author considers the possibility of using single-board computers in teaching disciplines related to parallel and distributed computing. It was revealed that the characteristics of the Raspberry Pi 3 Model B + are optimal in terms of price/quality ratio for the formation of an educational computing cluster for distributed and parallel computing. In [3], the principles of using decoys to detect attacks on Internet of Things devices were investigated. The use of the Raspberry $\mathrm{Pi}$ as a decoy for detecting malicious activity on the network has been proposed, it is justified that the use of the Raspberry Pi is a simple and effective solution to improve network security.

The topic of single-board computers and the future of the Internet of Things was studied in [4]. Also, a study was carried out of methods to increase the performance of architecture-independent platforms by means of parallelization of processes in works [5, 6].

When developing the method described in this work, the experience and results of works [7,8] were used. The materials explored faster algorithms that make better use of a fixed amount of processing power, and scalable algorithms that make the best use of large clusters of computers. The effectiveness and advantages of the implementation of these methods in the energy system were investigated by the authors of [9]. In the materials of works [10,11], the use of a single-board computer Raspberry Pi in systems to ensure network security is investigated and the use of methods to improve performance to ensure stable operation is substantiated.

However, taking into account the described scientific achievements on the research topic, the disclosure of the structure and principles of increasing the performance of the Raspberry Pi single-board computer remains open and requires detailed study.

So, the object of research is the Raspberry Pi singleboard computer. The aim of research is to reveal the structure and principles of increasing the performance of the Raspberry Pi single-board computer.

\section{Methods of research}

The study is based on an integrated scientific approach based on a systemanalytical, structural-functional, empirical and typological approach. Representations of the architectural composition of the Raspberry Pi single-board computer and typed principles of increasing productivity using a systems-analytical approach have been formed. An empirical approach has made it possible to investigate the genesis of single board computers.

\section{Research results and discussion}

The Raspberry Pi single board computer was developed for scientific purposes in 2012. Over time, the Raspberry Pi has gone through several modifica- tions, each of which differed from the previous version by changing one parameter. This approach made it possible to adjust the cost of the product depending on the needs of the user, which also had a positive effect on the popularity of the device. The entire Raspberry Pi line uses APM processors, which is a 32-bit RISC processor architecture. This is an energy-saving technology that is relevant in today's environment. In Fig. 1 shows the appearance of one of the popular Raspberry Pi B+boards.

In the information technology market, there are ten versions of the Raspberry Pi. The latest version is dated November 2020, it is equipped with wireless WiFi and Bluetooth modules ( $2 \times$ USB 3.0 and $1 \times$ USB 2.0 ports type A, 5.0, BLE), which expand the boundaries of miniPC application in the field of Ethernet technologies and has a frequency of $1.8 \mathrm{GHz}$.

The performance improvements of the Raspberry $\mathrm{Pi}$ single board computer can be achieved by adding ZRAM. ZRAMs are block devices based on compressed random access memory (RAM). The ZRAM module creates RAMbased block devices named $/ \mathrm{dev} / \mathrm{zram}<\mathrm{id}>(<\mathrm{Id}>=0,1, \ldots)$. Pages written to these discs are compressed and stored in memory itself. These drives provide very fast I/O information, and compression saves memory. Some of the use cases include/tmp storage, which can be used as additional platform drives. Statistics for individual ZRAM devices are exported via sysfs nodes to /Sys/block/zram $<\mathrm{id}>/$. When installing on the basis of Raspberry Pi ZRAM, the latter creates in RAM a block storage named / dev/ zram0 (or 1, 2, 3, etc.).

The recorded pages are compressed and stored in memory. ZRAM activation on the Raspberry Pi is shown in Fig. 2.

The next principle to improve the performance of the Raspberry Pi single board computer is the use of an NVMe disk. Non-Volatile Memory Express (NVMe) is a storage interface introduced in 2013 [13]. «Non-Volatile» means that the storage is not erased when the computer is rebooted, and «Express» means that data is transferred via the PCI Express (PCIe) interface on the computer's motherboard. This gives the drive a direct connection to the motherboard as data does not have to go through the SATA controller. Due to the fact that NVMe-disk is reliable and has a high data transfer rate, connecting it to a single-board computer Raspberry $\mathrm{Pi}$ is the optimal solution to improve performance.

40-pin header with UART, I2C, SPI, PWM interfaces, etc

$\downarrow$

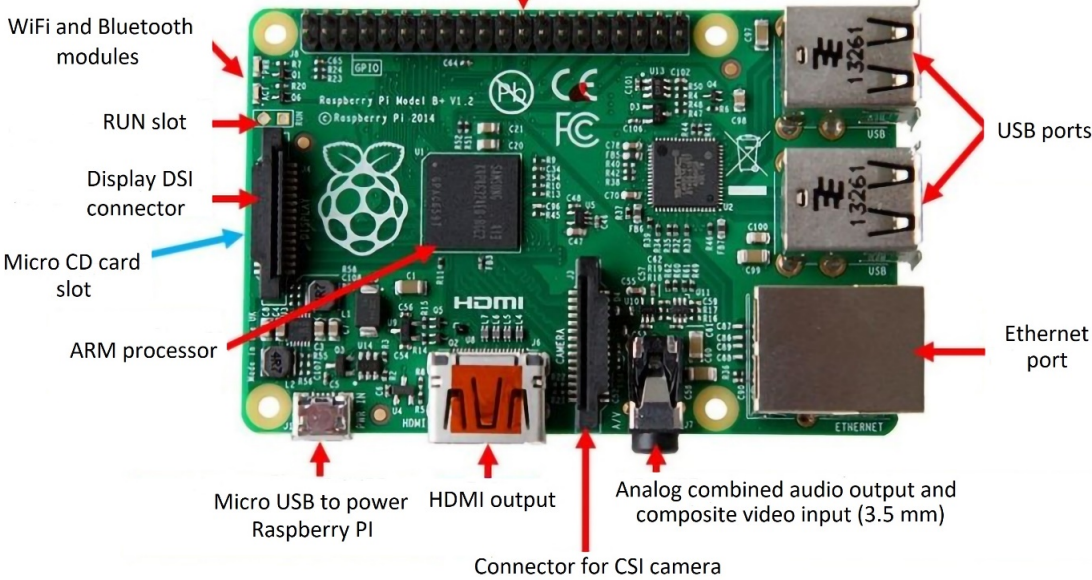

Fig. 1. External view of the Raspberry Pi board $B+[12]$ 


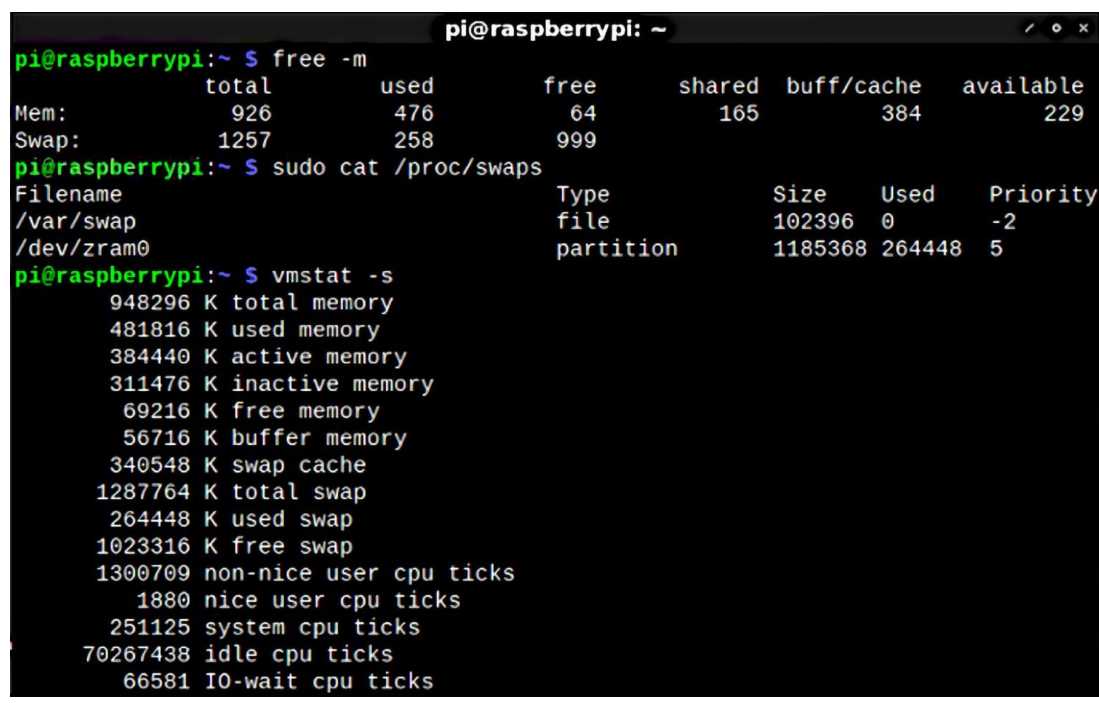

Fig. 2. Activating ZRAM on Raspberry $\mathrm{Pi}$

The NVMe disk is inserted into the adapter and connected to the Raspberry Pi. The setup sequence is as follows:

1. Writing the operating system (OS) to the SD card, creating the boot.txt file in Chapter 1, connecting the card to the Raspberry Pi and loading it.

2. Connecting to the board:

sshpi@raspberrypi.local.

3. System update:

sudo apt update -qy

$\& \&$ sudo apt upgrade -qy.

4. Enabling booting from USB drives:

Advanced Options $>$ Boot Order > USB Boot.

5. Changing the size of video memory from $65 \mathrm{MB}$ to $16 \mathrm{MB}$ through:

Options > GPU Memory.

6. USB NVMe disk and SD card connection to the computer using a card reader

7. Duplicate SD card to NVMe disk.

8. Booting the Raspberry Pi via USB and connecting to the board:

ssh pi@raspberrypi.local.

9. Installing hdparm to examine disk performance:

sudo apt install -qy hdparm.

10. Checking the speed of the disk (Fig. 3).

Thus, a new data store on the Raspberry Pi is obtained, which realizes the performance enhancement of the single board computer.
Fig. 3. The result of the NVMe-disk based on the Raspberry $\mathrm{Pi}$ single-board computer

The next principle to improve performance is to install the ICE Tower CPU. ICE Tower CPU is a cooling system that is designed to keep the Raspberry Pi cool.

After updating the firmware of the board and the operating system, it is necessary to:

1. edit/b00t/config.txt as root:

force turbo $=0$

arm_freq $=2000$

over_v0ltage $=6$

2. Reload the board.

3. Set the voltage to $1.03 \mathrm{~V}$ :

vcgencmd measure_v0lts

$\mathrm{v} 01 \mathrm{t}=1.03 \mathrm{~V}$

over_voltage_will decrease or increase the maximum voltage in $0.025 \mathrm{~V}$ increments. The range is -16 to 8 or 0.8 to $1.4 \mathrm{~V}$.

4. Start sbc bench.sh.

Thus, it will be possible to monitor the temperature using rpi_monitor (Fig. 4).

Thus, a performance improvement of $26 \%$ to $34 \%$ is obtained, which is basically in line with the expected theoretical improvement of $34 \%$. 


\begin{tabular}{lcccccccccc} 
Time & fake/real & \multicolumn{1}{c}{ load $\%$ cpu } & $\%$ sys & $\%$ usr & $\%$ nice & $\%$ io & $\%$ irq & Temp & VCore \\
$15: 11: 29:$ & $2000 / 2000 \mathrm{MHz}$ & 3.17 & $14 \%$ & $1 \%$ & $12 \%$ & $0 \%$ & $0 \%$ & $0 \%$ & $48.7^{\circ} \mathrm{C}$ & $1.0335 \mathrm{~V}$ \\
$15: 11: 52:$ & $2000 / 2000 \mathrm{MHz}$ & 3.28 & $85 \%$ & $2 \%$ & $83 \%$ & $0 \%$ & $0 \%$ & $0 \%$ & $52.1^{\circ} \mathrm{C}$ & $1.0335 \mathrm{~V}$ \\
$15: 12: 34:$ & $2000 / 2000 \mathrm{MHz}$ & 4.43 & $96 \%$ & $48 \%$ & $47 \%$ & $0 \%$ & $0 \%$ & $0 \%$ & $53.1^{\circ} \mathrm{C}$ & $1.0335 \mathrm{~V}$ \\
$15: 12: 54:$ & $2000 / 2001 \mathrm{MHz}$ & 4.21 & $83 \%$ & $3 \%$ & $79 \%$ & $0 \%$ & $0 \%$ & $0 \%$ & $52.6{ }^{\circ} \mathrm{C}$ & $1.0335 \mathrm{~V}$ \\
$15: 13: 37:$ & $2000 / 2000 \mathrm{MHz}$ & 4.96 & $95 \%$ & $40 \%$ & $54 \%$ & $0 \%$ & $0 \%$ & $0 \%$ & $52.1^{\circ} \mathrm{C}$ & $1.0335 \mathrm{~V}$ \\
$15: 13: 57:$ & $2000 / 2000 \mathrm{MHz}$ & 4.61 & $82 \%$ & $3 \%$ & $79 \%$ & $0 \%$ & $0 \%$ & $0 \%$ & $52.6^{\circ} \mathrm{C}$ & $1.0335 \mathrm{~V}$ \\
$15: 14: 39:$ & $2000 / 2000 \mathrm{MHz}$ & 4.92 & $95 \%$ & $37 \%$ & $57 \%$ & $0 \%$ & $0 \%$ & $0 \%$ & $52.1^{\circ} \mathrm{C}$ & $1.0335 \mathrm{~V}$
\end{tabular}

a

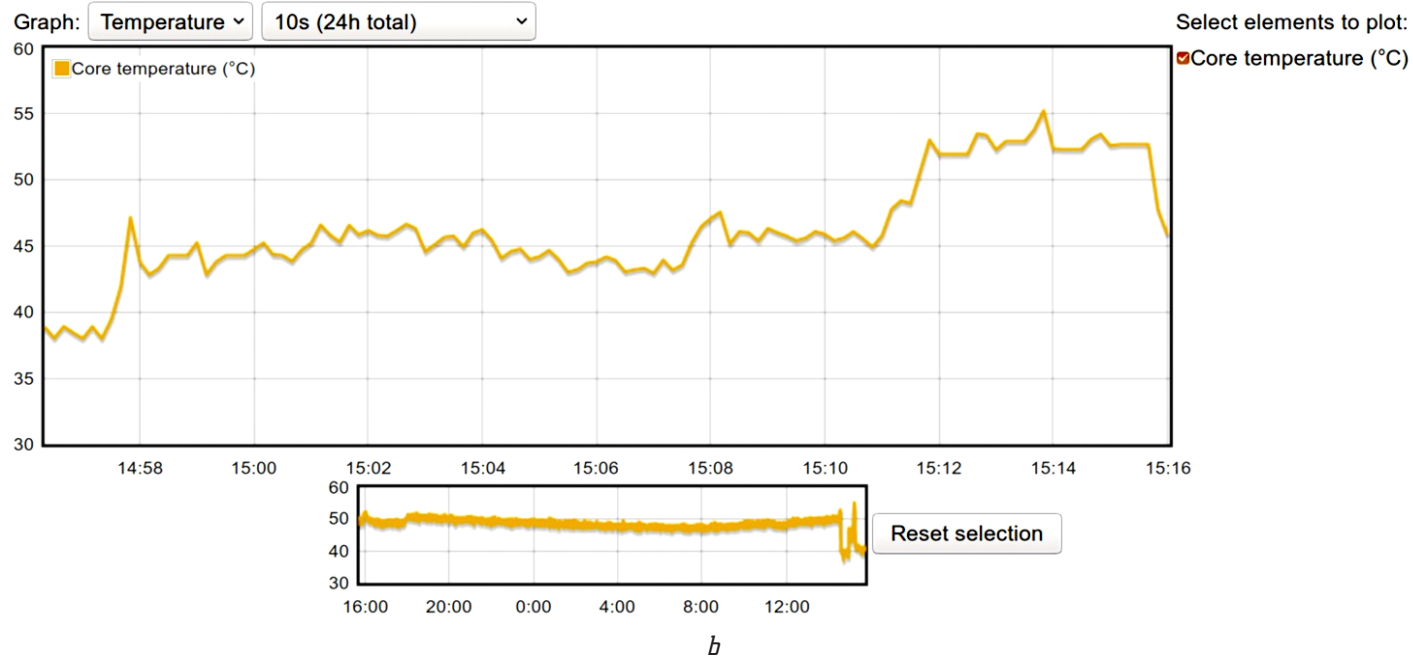

Fig. 4. Result of fluctuations in temperature components using грi_monitor: $a$ - summary table of temperature component results; $b$ - temperature dependence graph

\section{Conclusions}

The paper investigates the structure and describes a method for increasing the performance of a single-board computer using the example of Raspberry Pi. Based on the study, three main mechanisms for increasing performance are given, together they allow the board to solve problems that require high system performance (for example, to edit photos or videos), video surveillance recording, in turn, expands the range of applications. The performance improvement of the Raspberry Pi single board computer has been achieved through the addition of ZRAM. ZRAMs are block devices based on compressed random access memory (RAM). Pages written to these discs are compressed and stored in memory itself. These drives provide very fast $\mathrm{I} / \mathrm{O}$ information, and compression saves memory. The efficiency of adding a high-speed NVMe disk has been proven. The data transfer speed is increased in comparison with the usual microSD card $800 \mathrm{Mb} / \mathrm{s}$ and $90 \mathrm{Mb} / \mathrm{s}$, respectively. An increase in the performance of a single-board computer due to the installation of an ICE Tower CPU cooling system is investigated and a graph of the dependence of the load and temperature of the central processor is built. Prospects for further research are based on an increase in the speed of rendering imaging in comparison with conventional personal computers. This method of optimizing the operation of the Raspberry Pi single board computer is created using a combination of hardware and software optimization methods. The optimization method can be used not only for the Raspberry Pi single board computer, but also for many similar computers running Linux or systems built on the Linux kernel (Raspbian, Armbian, Ubuntu). Separate optimization methods have already been considered, but the combination of these methods to create a universal method for optimizing single board computers gives a performance increase from $26 \%$ to $34 \%$.

\section{References}

1. Babich, O., Boyko, Y., Galin, V., Chuprinsky, O. (2019). Design of intellectual information systems on the basis of MC Raspberry Pi. Electronics and Information Technologies, 11, 61-72. doi: http://doi.org/10.30970/eli.11.6

2. Sitsylitsyn, Y. (2019). Analysis of the use of Raspberry singlecircuit computers in the teaching of distributed and parallel computing. Scientific Papers of Berdiansk State Pedagogical University. Series: Pedagogical Sciences, 1, 92-99. doi: http://doi.org/ 10.31494/2412-9208-2019-1-1-92-99

3. Yatskiv, N. H., Byk, A. B., Mukomela, P. M., Bondar, V. M. (2020). Vykorystannia prymanok dlia vyiavlennia atak na prystroi Internet-rechei. Kiberbezpeka ta kompiuterno-infehrovani tekhnolohii (KBKIT - 2020). Ternopil, 26-28.

4. Lindqvist, U., Neumann, P. G. (2017). The future of the internet of things. Communications of the ACM, 60 (2), 26-30. doi: http://doi.org/10.1145/3029589

5. Franczak, T., Nkansahz, A., Marrinan, T., Papka, M. E. (2017). A Path from Serial Execution to Hybrid Parallelization for Learning HPC. Workshop on Education for High-Performance Computing ser. EduHPC'17. Denver.

6. Giacaman, N., Kalra, S., Sinnen, O. (2015). The active classroom: Students and instructors parallel programming in parallel. IEEE International Parallel and Distributed Processing Symposium Workshop. Piscataway, 739-745. doi: http://doi.org/10.1109/ ipdpsw.2015.24

7. Liu, J. (2016). 20 years of teaching parallel processing to computer science seniors. Workshop on Education for HighPerformance Computing (EduHPC). doi: http://doi.org/10.1109/ eduhpc.2016.006

8. Langmead, B. (2013). Practical software for big genomics data. IEEE 3rd International Conference on Computational Advances in Bio and medical Sciences (ICCABS). Orlando. doi: http:// doi.org/10.1109/iccabs.2013.6629241 
9. Papavasiliou, A., Oren, S. S., Rountree, B. (2015). Applying High Performance Computing to Transmission-Constrained Stochastic Unit Commitment for Renewable Energy Integration. IEEE Transactions on Power Systems, 30 (3), 1109-1120. doi: http://doi.org/10.1109/tpwrs.2014.2341354

10. Mahajan, S., Adagale, A. M., Sahare, C. (2016). Intrusion detection system using raspberry pi honeypot in network security. International Journal of Engineering Science and Computing, 6 (3), 2792-2795.

11. Razali, M. F., Razali, M. N., Mansor, F. Z., Muruti, G., Jamil, N. (2018). IoT Honeypot: A Review from Researcher's Perspective. 2018 IEEE Conference on Application, Information and Net- work Security (AINS). Langkawi. doi: http://doi.org/10.1109/ ains.2018.8631494

12. Obzor plat Raspberry Pi. Available at: https://3d-diy.ru/wiki/ arduino-platy/obzor-plat-raspberry-pi/

13. Chto takoe diski NVMe $i$ stoit li ikh pokupat (2019). Available at: https://guidepc.ru/articles/chto-takoe-diski-nvme-i-stoitli-ih-pokupat/

Liubomyr Duda, Postgraduate Student, Department of Robotics and Specialized Computer Systems, Cherkasy State Technological University, Cherkasy, Ukraine, e-mail: lyubomir-duda@ukr.net, ORCID: https://orcid.org/0000-0003-3218-3086 